IFT-UAM/CSIC-04-53

hep-th/0410162

\title{
Reduction of coupling constants in two-dimensional sigma models.
}

\author{
Enrique Álvarez \\ Instituto de Física Teórica UAM/CSIC, C-XVI, and Departamento de Física Teórica, \\ $C-X I$, \\ Universidad Autónoma de Madrid E-28049-Madrid, Spain
}

\begin{abstract}
Two-dimensional sigma models in curved target spaces are considered in which a relationship between the warp factor and the dilaton is imposed in a renormalization group invariant way.
\end{abstract}




\section{Introduction}

This paper deals with some aspects of two-dimensional quantum field theories of $d$ scalar fields $X^{\mu}\left(x^{a}\right)$ (where $1 \leq \mu, \nu, \ldots \leq d$ and $\left.1 \leq a, b, \ldots \leq 2\right)$ of the form:

$$
S=\frac{1}{4 \pi l_{s}^{2}} \int d^{2} x \sum_{a, b=1,2} g_{\mu \nu}(X) \partial_{a} X^{\mu} \partial_{b} X^{\nu} \gamma^{a b}
$$

(Here $l_{s}$ is an arbitrary length scale). The quantities $g_{\mu \nu}(X)$ can be viewed as twodimensional background fields, depending on the imbeddings $X^{\mu}\left(x^{a}\right)$,i.e., as generalized couplings. Two-dimensional sigma models are interesting for a variety of reasons. First of all, they obviously enjoy a beatiful geometrical intepretation in terms of embeddings of a two-dimensional manifold with metric $\gamma_{a b}$ in a target d-dimensional manifold with metric $g_{\mu \nu}$. This fact was successfully exploited by Friedan ([3] ) in order to compute the renormalization group ( $R G$ ) equations corresponding to the generalized couplings. In many cases, they enjoy physical phenomena like a mass gap, and have thus been studied as simplified versions of gauge theories.

If we supplement the lagrangian density with two other terms, namely

$$
i \epsilon^{a b} b_{\mu \nu}(X) \partial_{a} X^{\mu} \partial_{b} X^{\nu}+l_{s}^{2} R \Phi(X)
$$

corresponding to an antisymmetric two-dimensional background field, $b_{\mu \nu}(X)$, the KalbRamond field, and to another scalar two dimensional background field $\Phi(X)$, the dilaton, which couples to the two-dimensional scalar curvature, $R[\gamma]$, then the sigma model em-

bodies the universal massless fields of a string. The constant $l_{s}$ is then the string length, related to the famous $\alpha^{\prime}$ parameter through $\alpha^{\prime}=l_{s}^{2}$.

It is believed that consistency of the quantum string demands that this two-dimensional sigma model is Weyl invariant, which in turn implies the vanishing of the corresponding beta functions of the physical string fields, viewed as generalized two-dimensional couplings.

These beta functions are only known in the sigma model loop expansion, which physically is an expansion in powers of $\left(l_{s} / l\right)^{2}$, where $l$ sets the scale of curvature in the target 
manifold; that is, a low energy, low curvature expansion.

Recent analysis of Perelman [10], following previous ideas of Hamilton (cf. [8] for a review) of the problem of the classification of three-dimensional geometries draw on the analysis of precisely this RG equations, which mathematicians call the Ricci flow:

$$
\frac{\partial}{\partial t} g_{\alpha \beta} \equiv \mu^{-1} \frac{\partial}{\partial \mu^{-1}} g_{\alpha \beta}=-\beta_{\alpha \beta}\left(g_{\rho \sigma}\right)
$$

A very simple modification of the flow, due also to Hamilton, allows for another proof of the uniformization theorem for two dimensional geometries. The modification is

$$
\frac{\partial}{\partial t} g_{\alpha \beta}=-2 R_{\alpha \beta}+\frac{2 \pi \chi}{V} g_{\alpha \beta}
$$

where $V$ is the volume of the two-dimensional manifold

$$
V \equiv \int \sqrt{g} d^{2} x
$$

and $\chi$ the Euler characteristic

$$
\chi \equiv \frac{1}{2 \pi} \int \sqrt{g} R d^{2} x
$$

Hamilton [5] and others proved that the flow exists and converges to its fixed points, where

$$
R=\frac{2 \pi \chi}{V}
$$

which are precisely the constant curvature metrics. This modified flow is usually dubbed normalized for obvious reasons.

Another modification of the flow allows for arbitary target space diffeomorphisms along it; this amounts to the replacement:

$$
\begin{aligned}
& \beta_{\mu \nu}\left(g_{\alpha \beta}\right) \rightarrow \beta_{\mu \nu}\left(g_{\alpha \beta}\right)+\nabla_{\mu} \xi_{\nu}+\nabla_{\nu} \xi_{\mu} \\
& \beta(\Phi) \rightarrow \beta(\Phi)+\xi^{\mu} \nabla_{\mu} \Phi
\end{aligned}
$$

The fixed points of this flow; that is, the fixed points of the ordinary flow up to diffeomorphisms, are called Ricci solitons. 
What we want to explore here is the possibility that there are reductions of coupling constants (studied in the standard model of particle physics by Hill ([ 6$]$ ) and Zimmermann $([14]))$; that is, a hypersurface in parameter space which is left invariant by the RG flow. In order to determine them, it is clearly necessary to study the sigma model away from its conformal fixed point.

In the absence of $b$-field (just by simplicity), we shall assume a reduction of the form

$$
g_{\mu \nu}(X)=g_{\mu \nu}(\Phi(X))
$$

that is, the metric expressed in terms of the dilaton. This makes physical sense, because the dilaton is related to the string coupling constant, $g_{s}$.

Consistency of the RG flow to one loop in the sigma model expansion is achieved when

$$
R_{\mu \nu}+\left.2 \nabla_{\mu} \nabla_{\nu} \Phi\right|_{g_{\mu \nu}=g_{\mu \nu}(\Phi)}=\left.\frac{d g_{\mu \nu}}{d \Phi}\left(\Delta-\frac{1}{2} \nabla^{2} \Phi+g^{\mu \nu} \nabla_{\mu} \Phi \nabla_{\nu} \Phi\right)\right|_{g_{\mu \nu}=g_{\mu \nu}(\Phi)}
$$

It is tempting to write

$$
\Delta \equiv \frac{d-26}{6 l_{s}^{2}}
$$

as seems to be indicated by the flat space limit, but we would like to keep an open mind on this, thinking on the curved Liouville interpretation, and we shall treat it as a free parameter.

This indeed guarantees that the functional relationship is a first integral of the RG equations.

\section{The flow in warped compactifications.}

The essential property of the two-dimensional RG flow is that it is irreversible, at least when the target space is compact (cf. [13]). This is guaranteed by Zamolodchikov's theorem, which actually ensures that there is a monotonically decreasing function (the c-function) defined on the flow, and which equals the central charge at the conformal points. Much less 
is know for noncompact spaces, although recent progress has been reported ([9]) amounting for instance to the absence of breather modes.

In order to get some intuition in some physically interesting examples, we shall study the metric

$$
d s^{2}=d \rho^{2}+a(\rho) \delta_{i j} d x^{i} d x^{j}
$$

This metric is the simplest instance of those which admit in adequate circumstances a holographic interpretation (cf. [1], and [1] for conformal invariant examples). The field $\rho$, can be thought of as the Liouville mode, and the warping factor $a(\rho)$ as due to the peculiar measure associated to the Liouville field.

The RG flow is given by:

$$
\begin{aligned}
& \frac{1}{l_{s}^{2}} \frac{\partial g_{\rho \rho}}{\partial \log \mu}=\left(\frac{a^{\prime}}{a}\right)^{2}-2 \frac{a^{\prime \prime}}{a}+2 \Phi^{\prime \prime}=0 \\
& \frac{\mu}{l_{s}^{2}} \frac{\partial a}{\partial \mu} \delta_{i j}=-\frac{1}{2}\left(a^{\prime \prime}+\frac{\left(a^{\prime}\right)^{2}}{a}\right) \delta_{i j}+a^{\prime} \Phi^{\prime} \delta_{i j} \\
& \frac{\mu}{l_{s}^{2}} \frac{\partial \Phi}{\partial \mu}=\Delta-\frac{1}{2}\left(\Phi^{\prime \prime}+\frac{d-1}{2} \frac{a^{\prime}}{a} \Phi^{\prime}\right)+\left(\Phi^{\prime}\right)^{2}
\end{aligned}
$$

where $a^{\prime} \equiv \frac{d a}{d \rho}$ and $\Phi^{\prime} \equiv \frac{d \Phi}{d \rho}$. It has been kept $g_{\rho \rho}=1$ along the flow, which means more or less imposing a coordinate gauge condition on it.

The RG equations (13) do not in general keep invariant a given ansatz, and the whole system of $d(d+1) / 2$ gravitational $g_{\mu \nu}$ variables, plus $d(d-1) / 2$ antisymmetric $b_{\mu \nu}$, plus the dilaton $\Phi$, that is, a grand total of $d^{2}+1$ background fields, is excited with generic initial conditions.

In some particular cases, however, it is consistent to assume that some of those fields vanish, or else keep a simple functional dependence on the imbeddings. 


\subsection{Perturbing the anti-de Sitter background}

Let us for example study now in detail (euclidean) anti de-Sitter (adS) geometry, characterized by:

$$
a(\rho)=e^{-\frac{2 \rho}{l}}
$$

The first equation of the set (13) then implies that

$$
\Phi=\left(\frac{\rho}{l}+\phi\right)^{2}
$$

(where $\phi$ is a constant) thus completely determining the dilaton. The two remaining equations are consistent only when

$$
\Delta=\frac{1}{l^{2}}
$$

and

$$
d=5
$$

In case $\Delta \equiv \frac{d-26}{6 l_{s}^{2}}$ the radius of adS is given through:

$$
\frac{l^{2}}{l_{s}^{2}}=\frac{6}{d-26} .
$$

which means that the total dimension must exceed 26. This can be achieved by adding enough spectator dimensions.

At any rate, the $\mathrm{RG}$ equations then reduce to

$$
\frac{\mu}{l_{s}^{2}} \frac{\partial \rho}{\partial \mu}=\frac{2}{l}\left(1+\phi+\frac{\rho}{l}\right)
$$

which integrates to

$$
\frac{\rho}{l}=\left(\frac{\mu}{\mu_{0}}\right)^{\frac{2 l_{s}^{2}}{l^{2}}}-1-\phi
$$

This leads to the identification of the ultraviolet (UV) region, in the two-dimensional RG sense, with the region where $\rho \rightarrow \infty$, or what is the same, $a \rightarrow 0$. 


\subsection{Perturbations of the confining background}

Let us now consider perturbations of the confining background of [1]. In the present notation this is given by:

$$
\begin{aligned}
& a(\rho)=\rho+\delta a(\rho) \\
& \Phi(\rho)=\frac{1}{2} \log (\rho)+\delta \Phi(\rho)
\end{aligned}
$$

Let us insist again on the fact that it is not guaranteed by any means that there are perturbations with such a simple dependence on the target space coordinates, and with only those background field excited. In our case, the first equation of (13) imposes at the linearized level the restriction

$$
\delta a^{\prime \prime}+\frac{\delta a}{\rho^{2}}-\frac{\delta a^{\prime}}{\rho}-\rho \delta \Phi^{\prime \prime}=0
$$

Now the other two equations yield two equations for the flow of the two-dimensional field $\rho$, which for consistency has to be of $\mathcal{O}(1)$, that is, of the same order of the perturbation:

$$
\begin{aligned}
& \frac{1}{l_{s}^{2}} \frac{\partial \rho}{\partial \log \mu}=-\frac{\delta a^{\prime \prime}}{2}-\frac{\delta a^{\prime}}{2 \rho}+\frac{\delta a}{2 \rho^{2}}+\delta \Phi^{\prime} \\
& \frac{1}{l_{s}^{2}} \frac{\partial \rho}{\partial \log \mu}=-\frac{\delta a^{\prime}}{\rho}+\frac{\delta a}{\rho^{2}}-\rho \delta \Phi^{\prime \prime}
\end{aligned}
$$

Consistency of the posited ansatz fully determines the allowed perturbations to be:

$$
\begin{aligned}
& \delta a=2 c_{1}+c_{2} \rho+c_{3} \rho \log (\rho) \\
& \delta \Phi=c_{4}+\frac{c_{1}}{\rho}
\end{aligned}
$$

Under these circumstances, many terms cancel in the equations, in such a way that the

flow is completely independent of the perturbation of the dilaton, and is only sensitive to the $\rho \log (\rho)$ piece of the perturbation of the metric. The constant $c_{3}$ has to be negative, and the equation can be easily integrated to yield

$$
\rho \sim\left(\log \left(\mu / \mu_{0}\right)\right)^{1 / 2}
$$

so that this time we obtain the opposite behavior to the previous example; the UV region corresponds to the direction of growing warping factors. 


\section{Reduction of coupling constants in warped com- pactifications.}

Let us now finally examine the possibility that all two-dimensional coupling constants, such as the spacetime metric, are functions of one amongst them, for example the dilaton.

First of all, in all consistent perturbations with some symmetry, such as the ones considered in the previous paragraph, this is always so: given the functions $a(\rho)$ and $\Phi(\rho)$ one can always construct the function $a(\Phi)$. What we want to do here is to give a direct construction of the general situation.

$$
\begin{aligned}
& \frac{1}{l_{s}^{2}} \frac{\partial g_{\rho \rho}}{\partial \log \mu}=\left(\frac{a^{\prime}}{a}\right)^{2}-2 \frac{a^{\prime \prime}}{a}+2 \Phi^{\prime \prime} \\
& \frac{1}{l_{s}^{2}} \frac{\partial a}{\partial \log \mu} \delta_{i j}=-\frac{1}{2}\left(a^{\prime \prime}+\frac{\left(a^{\prime}\right)^{2}}{a}\right) \delta_{i j}+a^{\prime} \Phi^{\prime} \delta_{i j} \\
& =\frac{d a}{d \Phi}\left(\Delta-\frac{1}{2}\left(\Phi^{\prime \prime}+\frac{d-1}{2} \frac{a^{\prime}}{a} \Phi^{\prime}\right)+\left(\Phi^{\prime}\right)^{2}\right) \delta_{i j}
\end{aligned}
$$

Let us consider reduced flows starting from adS. We shall consider $\frac{d a}{d \Phi}=\frac{a^{\prime}}{\Phi^{\prime}}$, although more general situations can be examined, in which the background fields depend in a nontrivial way on all their arguments. The second equation of the set (26), which is the nontrivial equation guaranteeing the fact that the constraint is a first integral of the RG flow, reduces to

$$
\Phi^{\prime \prime}+\frac{5-d}{l} \Phi^{\prime}-2 \Delta=0
$$

which can be easily solved when $d \neq 5$ to yield:

$$
\Phi=A+\frac{2 l \Delta}{5-d} \rho+B e^{-\frac{5-d}{l} \rho}
$$

where $A$ and $B$ are arbitrary dimensionless constants. This is equivalent to the functional relationship

$$
\Phi=-\frac{l^{2} \Delta}{5-d} \log a-B a^{\frac{5-d}{2}}+A
$$


When $d=5$ instead,

$$
\Phi=\Delta \rho^{2}+C_{1} \frac{\rho}{l}+C_{2}
$$

where $C_{1}$ and $C_{2}$ are dimensionless constants.

The flow is now determined (again, when $d \neq 5$ ) through

$$
\frac{\partial \rho}{\partial \log \mu}=\alpha+\beta e^{-\gamma \rho}
$$

with $\alpha \equiv \frac{2 l_{s}^{2}}{l}+\frac{2 l \Delta l_{s}^{2}}{5-d}, \quad \beta \equiv \frac{d-5}{l} B l_{s}^{2}$ and $\gamma \equiv \frac{5-d}{l}$

The solution is

$$
e^{-\gamma \rho}=\frac{\alpha}{D l\left(\mu / \mu_{0}\right)^{\alpha \gamma}-\beta}
$$

where $D$ is a new dimensionless constant of integration.

The character of the flow depends on the signs appearing in this equation. For example $\alpha \gamma=2 \frac{l_{s}^{2}}{l^{2}}\left(5-d+l^{2} \Delta\right) \sim 2 l_{s}^{2} \Delta$ if the curvature is small in string units.

There are two types of behavior: either

$$
e^{-\gamma \rho} \sim \frac{1}{\left(\mu / \mu_{0}\right)^{\left|C_{1}\right|} \pm\left|C_{2}\right|}
$$

(with $C_{1}$ and $C_{2}$ arbitrary constants) which tends to a constant in the IR, and to zero in the UV, when the sign in the denominator is positive, or to a Landau pole with the negative sign.

The other possible behavior is

$$
e^{-\gamma \rho} \sim \frac{1}{\left(\mu / \mu_{0}\right)^{-\left|C_{1}\right|} \pm\left|C_{2}\right|}
$$

tending to a constant in the UV and to 0 in the IR, again with Landau poles appearing when the sign in the denominator is negative.

Finally, in the special case $d=5$, the flow is

$$
\frac{\rho}{l}=E+F\left(\frac{\mu}{\mu_{0}}\right)^{2 \Delta l^{2}}
$$


withe arbitrary dimensionless constants $E$ and $F$. When $\Delta<0$ both the UV and IR limits are then $\rho=$ constant, which can again be tuned to keep $g_{\rho \rho}$ bounded.

\subsection{Constrained flow}

Alternatively, it is possible to impose the constraint that $g_{\rho \rho}=1$ along the flow. The relevant equations are in this case:

$$
\begin{aligned}
& \left(\frac{a^{\prime}}{a}\right)^{2}-2 \frac{a^{\prime \prime}}{a}+2 \Phi^{\prime \prime}=0 \\
& -\frac{1}{2}\left(a^{\prime \prime}+\frac{\left(a^{\prime}\right)^{2}}{a}\right) \delta_{i j}+a^{\prime} \Phi^{\prime} \delta_{i j}=\frac{d a}{d \Phi}\left(\Delta-\frac{1}{2}\left(\Phi^{\prime \prime}+\frac{d-1}{2} \frac{a^{\prime}}{a} \Phi^{\prime}\right)+\left(\Phi^{\prime}\right)^{2}\right) \delta_{i j}
\end{aligned}
$$

In our simple setting it is still true that

$$
\frac{d a}{d \Phi}=\frac{a^{\prime}}{\Phi^{\prime}}
$$

We shall take the following point of view. Given the dilaton, $\Phi(\rho)$, the warp factor is determined by the first equation to be:

$$
a=a_{0} e^{\int^{\rho} f(x) d x}
$$

where the auxiliary function $f(x)$ is a solution of the Riccati differential equation:

$$
f^{\prime}+\frac{f^{2}}{2}=\Phi^{\prime \prime}
$$

We shall write

$$
f[\Phi]
$$

to emphasize the fact that this function is actually fully determined, once the dilaton $\Phi$ is known.

The remaining equation is then a very implicit one for the dilaton:

$$
\Phi^{\prime}\left(\frac{f^{\prime}[\Phi]}{2}+\frac{5-d}{4} f[\Phi]^{2}\right)-\frac{1}{2} f[\Phi] \Phi^{\prime \prime}+\Delta f[\Phi]=0
$$


It is remarkable that a linear dilaton demands $\Delta=0$ and besides, $d=4^{2}$. The first equation (39) then gives $f \sim \frac{2}{\rho}$, that is,

$$
a=a_{0}\left(\rho / \rho_{0}\right)^{2} \sim \Phi^{2}
$$

It is clear that a whole taxonomy of reduced flows can be constructed along these lines.

What is more important from the physical point of view is whether there is uniformization of the two-dimensional RG flow for arbitrary dimension of the target space. This would mean that the sigma model evolves (in the IR) towards the conformal fixed point, and if the reduction of coupling constants holds all the way during the flow, it should also hold in the conformal limit.

\section{Concluding remarks}

We have already mentioned the remarkable fact that the two dimensional sigma model RG evolution equations allow for a different proof of the classification theorem of twodimensional closed surfaces, and is currently believed to be the main ingredient towards the proof of Thurston's geometrization conjecture in three dimensions. Although this problem has been analyzed from a physicist'viewpoint in [4] 2], uncovering interesting relationships with integrable models of the Toda family in the two-dimensional case, it is likely that many more fascinating relationships between mathematics and physics lie hidden beneath the surface.

On the other hand, it is not clear what is the relationship of these models with string theory.

Critical strings corresponds to conformal points, and even non-critical (Liouville) strings are usually assumed to correspond to conformal models, albeit with non-trivial background fields.

\footnotetext{
${ }^{2}$ The apparent contradiction between these two statements when considering critical strings can again be resolved by introducing spectator dimensions.
} 
Many efforts have been devoted over the years to find a string representation of a gauge theory. The main success up to date has been the adS/CFT conjecture of Maldacena [7. Although this particular example corresponds to a non-confining theory, simple modifications of the original correspondence are able to represent confinement, to a certain extent 12. In all these examples, the string dealt with is a critical string.

It is not unthinkable, however, that some relationship exists between the four-dimensional $\mathrm{RG}$ flow of a non supersymmetrical gauge theory and the two-dimensional RG flow of some sigma-model related to the hypothetical confining string.

The simplest of those models, (such as the ones in [1]) in which a direct relationship is posited between the gravitational degrees of freedom and the gauge coupling constant (determined by the string coupling constant, which is in turn determined by the dilaton) are the ones in which a reduction of two-dimensional coupling constants is made, somewhat similar to the ones introduced in this paper.

More work is clearly needed in this facinating topic, both by itself, and by its possible relationship with the confining string.

\section{Acknowledgments}

I am indebted to Jorge Conde for a careful reading of the manuscript. This work has been partially supported by the European Commission (HPRN-CT-200-00148) and by FPA2003-04597 (DGI del MCyT, Spain).

\section{References}

[1] E. Alvarez and C. Gomez, "The renormalization group approach to the confining string," Nucl. Phys. B 574 (2000) 153 arXiv:hep-th/9911215. 
[2] I. Bakas, "Renormalization group flows and continual Lie algebras," JHEP 0308 (2003) 013 arXiv:hep-th/0307154.

I. Bakas, "Ricci flows and infinite dimensional algebras," Fortsch. Phys. 52, 464 (2004) arXiv:hep-th/0312274.

I. Bakas, "Ricci flows and their integrabillity in two dimensions" arXiv:hep-th/0410093.

[3] D. Friedan, "Nonlinear Models In Two Epsilon Dimensions," Phys. Rev. Lett. 45 (1980) 1057.

[4] J. Gegenberg and G. Kunstatter, "Using 3D stringy gravity to understand the Thurston conjecture," Class. Quant. Grav. 21 (2004) 1197 arXiv:hep-th/0306279.

J. Gegenberg and G. Kunstatter, "Ricci Flow of 3D Manifolds with One Killing Vector" arXiv:hep-th/0409283.

[5] R.S. Hamilton,"The Ricci flow on surfaces", Contemp. Math. 71 (1988) 237.

[6] C. T. Hill, "Quark And Lepton Masses From Renormalization Group Fixed Points," Phys. Rev. D 24 (1981) 691.

[7] J. M. Maldacena, "The large N limit of superconformal field theories and supergravity," Adv. Theor. Math. Phys. 2 (1998) 231 [Int. J. Theor. Phys. 38 (1999) 1113] arXiv:hep-th/9711200.

[8] J. Milnor, "Towards the Poincaré conjecture and the classification of 3-Manifolds" Notices of the AMS, 50,1226.

[9] T Oliynyk, V Suneeta, E Woolgar, "Irreversibility of World-sheet Renormalization Group Flow" arXiv:hep-th/0410001

[10] Grisha Perelman, "The entropy formula for the Ricci flow and its geometric applications", [arXiv:math:DG/0211159] 
[11] A. M. Polyakov, "Gauge fields and space-time," Int. J. Mod. Phys. A 17S1 (2002) 119 arXiv:hep-th/0110196.

[12] E. Witten, "Anti-de Sitter space, thermal phase transition, and confinement in gauge Adv. Theor. Math. Phys. 2 (1998) 505 arXiv:hep-th/9803131.

[13] A. B. Zamolodchikov, "Irreversibility' Of The Flux Of The Renormalization Group In A 2-D Field JETP Lett. 43 (1986) 730 [Pisma Zh. Eksp. Teor. Fiz. 43 (1986) 565].

[14] W. Zimmermann, "Reduction In The Number Of Coupling Parameters," Commun. Math. Phys. 97 (1985) 211. 